\title{
Are metallic stents really safe? A long-term analysis in lung transplant recipients
}

\author{
J. Gottlieb*, T. Fuehner*, M. Dierich*, O. Wiesner*, A.R. Simon* and T. Welte*
}

ABSTRACT: Airway complications affect $20 \%$ of all lung transplant recipients. Self-expandable metallic stents (SEMS) are one treatment option but their use in benign airway disorders is controversial. We studies the long-term safety of SEMS in lung transplant recipients.

Between January 1998 and February 2008, all lung transplant recipients with SEMS were analysed retrospectively at a single centre. Complications were recorded until September 2008. In $65(9.2 \%)$ out of 706 recipients, 111 (91\% noncovered) bronchial SEMS were implanted a median (range) 133 (55-903) days after lung transplantation; follow-up was 777 (7-3.655) days. Clinical improvement was noted in $80 \%$ of recipients. The forced expiratory volume in $1 \mathrm{~s}$ increased by (mean \pm SD) $21 \pm 33 \%$.

Most frequent early complications were migration (3\%) and mucus plugging (11\%). No procedure-related deaths were noted. Re-stenosis occurred in 34 (52\%) out of 65 recipients 85 (7629) days after insertion. In multivariate analysis, stent insertion before post-operative day 90 was independently associated with an increased risk of re-stenosis (HR 3.29, 95\% Cl 1.50-7.18; $p=0.003$ ). In $40 \%$ of recipients, new bacterial airway colonisation occurred after SEMS insertion. In SEMS patients, 5-yr survival was significantly lower than in the total cohort $(60 \%$ versus $76 \%$; $\mathrm{p}=0.02)$.

Late complications in lung transplant recipients treated with SEMS are frequent. The major problems are re-stenosis and airway colonisation.

KEYWORDS: Airway obstruction, bronchiolitis obliterans syndrome, granulation tissue, lung transplantation, prosthesis infections, stents

A irway complications are a continuing problem following lung transplantation and can be divided into necrotic or obstructive lesions [1]. Approximately $20 \%$ of lung transplant recipients are affected [1-3]. Necrotic lesions frequently heal spontaneously, with surgical repair rarely being required. In symptomatic obstructive lesions, suggested interventional techniques include balloon dilatation [4] and desobliteration, e.g. laser application, argon plasma coagulation [5], brachytherapy [6] and stent insertion [7].

Repeated balloon dilatation is recommended as first-line therapy. Up to $26 \%$ of lung transplant recipients with airway stenosis respond to single balloon dilatation [4], thermal desobliteration is effective but has a high recurrence rate [5]. Both techniques allow time for the lesion to mature into a fibrous stricture, which is more suitable for stent placement.

Classically, placement of a stent or prosthesis is indicated in case of external compression [8] of the airway, but stents in lung transplant recipients are frequently used in recurrent endoluminal lesions as well. A variety of different stent models have been commercially available over the last 20 yrs. Silicone stents are rarely used in post-lung transplant patients due to their decreased inner diameter, worse adaptability when target lesions are complex or angulated, and the frequent need for ventilation through interstices in this cohort. Most experience in lung transplantation has been gained with self-expanding metallic stents (SEMS). Successful insertion of SEMS with flexible bronchoscopy under and, increasingly, without general anaesthetic has been increasingly reported [9]. However, concern remains about the safety of metallic stents in treating benign tracheal lesions [10]. Complications such as migration, bleeding, atelectasis, infection, re-stenosis and fracture have been reported.

SEMS consist of a self-expanding, knitted metallic mesh usually made from nitinol, a nickel-titanium alloy [11]. The knitted design of these stents permits axial and radial movements of the wire filaments, which allows excellent adaptation and prevents longitudinal expansion of the stent when
AFFILIATIONS

*Depts of Pulmonary Medicine, and

\# Cardiothoracic, Transplantation and Vascular Surgery, Hanover Medical School, Hanover, Germany.

CORRESPONDENCE

J. Gottlieb

Dept of Respiratory Medicine OE6870

Hanover Medical School

Carl-Neuberg-Str. 1

30625 Hanover

Germany

E-mail: gottlieb.jens@mh-

hannover.de

Received:

March 152009

Accepted after revision:

April 172009

First published online:

May 142009
European Respiratory Journal Print ISSN 0903-1936 Online ISSN 1399-3003 
compressed. Despite being commercially available for $>10$ yrs, literature regarding the long-term outcome of SEMS in lung transplant recipients remains sparse.

The aim of this study was to assess the long-term complications and survival in patients with airway complications after lung transplantation following insertion of a single type of SEMS.

\section{METHODS}

\section{Study design}

A retrospective cohort study was performed at Hanover Medical School (Hanover, Germany) between January 1, 1998 and February 28, 2008. Inclusion criteria were patients undergoing lung transplantation and technically successful insertion of SEMS with follow-up of at least 7 days. Patient demographics are shown in table 1.

Follow-up was performed until September 30, 2008. The primary outcome was the occurrence of in-stent re-stenosis. Secondary outcomes included graft survival and bronchiolitis obliterans syndrome (BOS)-free survival.

Prior to enrolment, the study had been approved by the Institutional Review Board (Hanover). We did not receive any financial support from the pharmaceutical industry or any manufacturer of medical supplies.

\section{Procedures}

Prior to stent insertion, all patients underwent evaluation by fibreoptic bronchoscopy (FOB). Criteria for stent insertion were symptomatic (e.g. dyspnoea, infection, cough, haemoptysis and deteriorating graft function) obstructive lesions with a diameter of $<5 \mathrm{~mm}$ and recurrence after repeated balloon dilatation or desobliteration (e.g. argon plasma coagulation) or extensive anastomotic dehiscence.

\begin{tabular}{lc} 
TABLE 1 Patient demographics & \\
Subjects & 65 \\
Age yrs & $48(17-64)$ \\
Sex & \\
$\quad$ Female & $25(35)$ \\
Male & $40(65)$ \\
Baseline FEV $\mathbf{1} \%$ pred & $77 \pm 20$ \\
Time since transplantation at first stent days & $133(50-903)$ \\
Number of stents per patient & $1(1-7)$ \\
Underlying disease & \\
Emphysema & $30(46)$ \\
Cystic fibrosis & $13(20)$ \\
Pulmonary fibrosis & $13(20)$ \\
Pulmonary artery hypertension & $1(2)$ \\
Other & $8(12)$ \\
Procedure & \\
Single lung transplant & $2(3)$ \\
Double lung transplant & $61(94)$ \\
Heart-lung transplant & $2(3)$ \\
Follow-up after stent insertion days & $777(7-3.655)$ \\
\hline
\end{tabular}

Data are presented as $n$, median (range), mean \pm SD or $n(\%)$. FEV1: forced expiratory volume in $1 \mathrm{~s} ; \%$ pred: \% predicted.
The commercially available Ultraflex ${ }_{\circledR}$ stent (Boston Scientific, Natick, MA, USA) was used in all patients. Stent insertion was performed by flexible bronchoscopy under general anaesthetic or with moderate sedation, as previously described [12].

Before stent placement, airway recanalisation was performed by argon coagulation or balloon dilatation as needed. Suitable stent length was estimated by withdrawing the bronchoscope from the distal to the proximal end of the lesion and diameter was estimated by direct vision. Stents were deployed from its delivery catheter under direct vision without fluoroscopy and subsequent balloon dilatation was performed to allow full stent expansion.

\section{Follow-up}

Chest radiographs were performed on the same day or following day to evaluate stent position. Spirometry data were collected directly before and 4 days after stent placement. Spirometry, with measurements of forced expiratory volume in $1 \mathrm{~s}$ (FEV1) and forced vital capacity (FVC), were performed according to American Thoracic Society/European Respiratory Society guidelines [13].

All patients received nebulised saline after stent insertion to prevent stent obstruction by secretions. To prevent migration for 3 days after the procedure, patients received anti-tussive agents and were advised not to perform home spirometry.

All lung transplant recipients received frequent, individual, centre-based, life-long follow-up care. Patients were usually seen in our out-patient clinic (Hanover Medical School) every 6 weeks. Patients were instructed to use home spirometry daily and to contact the transplant centre in case of decline. In the case of acute unexplained respiratory symptoms, prompt attendance at our follow-up clinic was arranged. In case of symptoms, functional impairment or radiological abnormalities, repeat FOB was performed.

\section{Definitions}

Successful treatment was defined by improved clinical symptoms and/or an FEV1 increase of $\geqslant 10 \%$ from pre-treatment level. Re-stenosis was defined as the inability to pass a standard $4.9 \mathrm{~mm}$ bronchoscope (BF-P40 or BF-P180; Olympus, Tokyo, Japan). Complications were defined as early in stent-related clinical events occurring $\leqslant 30$ days after insertion, with late complications relating to any event thereafter.

BOS was defined as an FEV1 $<80 \%$ compared with baseline FEV1; defined as the average of the two highest measurements obtained $\geqslant 3$ weeks apart during the post-operative course [14]. Graft loss was defined as death or redo-transplantation.

Antibiograms of all isolates from lower respiratory tract samples (bronchoalveolar lavage (BAL) in the majority) were identified by standard laboratory methods before and after stent insertion. Airway colonisation of the graft was defined by repeated detection on at least two occasions regardless of the signs of infection.

\section{Statistical analysis}

Data are reported as mean \pm SD and time dependent variables are expressed as median (range). All reported p-values are 
two-sided, unless otherwise indicated. For all analyses, pvalues $<0.05$ were considered to be statistically significant.

Categorical variables were analysed by the Chi-squared test or Fisher's exact test, the log-rank test was applied to compare restenosis free survival. Medians were compared with the Mann-Whitney test and means were analysed with an unpaired t-test. Multivariate analysis included Cox stepwise forward regression analysis for re-stenosis free survival. All variables with a p-value $\leqslant 0.10$ were included and variables with a p-value of $>0.10$ were excluded in a multivariate analysis. Kaplan-Meier curves were plotted to compare overall and BOS-free survival in patients with or without re-stenosis.

\section{RESULTS}

During the 10-yr observation period, 706 lung transplant procedures involving 1,276 airway anastomoses were performed. In 65 (9.2\%) out of 706 lung transplant recipients, 111 SEMS were implanted.

\section{Bronchoscopic technique}

All stents were placed using flexible bronchoscopy. General anaesthetic was used in $48(72 \%)$ out of 65 patients, and insertion was performed via laryngeal mask in $13(20 \%)$ out of 65 patients. Noncovered stents were used in 59 (91\%) out of 65 patients and covered stents in six patients. The most frequently used stent length was $20 \mathrm{~mm}(\mathrm{n}=77)$, while $30 \mathrm{~mm}(\mathrm{n}=26)$ and $40 \mathrm{~mm}(\mathrm{n}=8)$ lengths were chosen less frequently. The stent diameters used were $10 \mathrm{~mm}(\mathrm{n}=84), 8 \mathrm{~mm}(\mathrm{n}=20)$ and $12 \mathrm{~mm}$ $(n=7)$. In $25(38 \%)$ out of 65 study subjects, multiple stents were inserted (maximum seven per patient). In 16 (25\%) out of

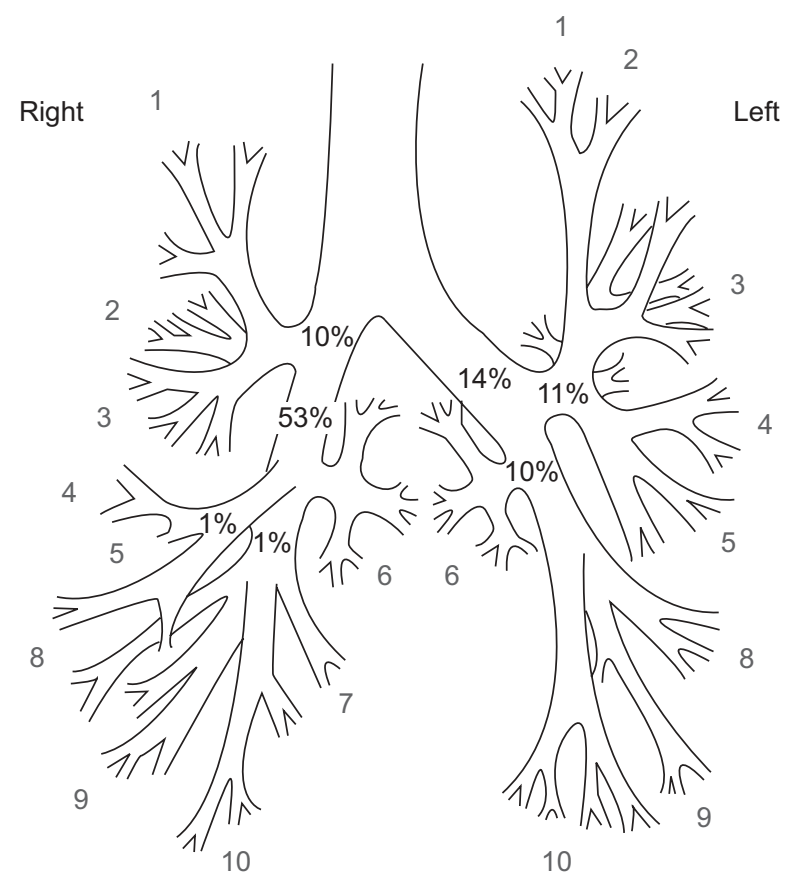

FIGURE 1. Target lesions for stent insertion $(n=111)$ : intermediate bronchus $(n=59)$, left main bronchus $(n=16)$, right upper lobe bronchus $(n=11)$, left upper lobe bronchus $(n=12)$, middle lobe bronchus $(n=1)$, right lower lobe bronchus $(n=1)$, left lower lobe bronchus $(n=11)$
65 patients, multiple target lesions were approached. In 12 patients, bilateral SEMS were implanted.

Insertion was performed 133 (50-903) days post surgery. In $72 \%$ of patients, the first stent was placed within the first 6 months after lung transplantation. Indications for stent insertion were obstructive lesions in all but one patient who had a pure necrotic lesion.

Target lesions for stent insertion are shown in figure 1. In 76\% of patients, the post-anastomotic region was involved rather than the anastomosis and there was predominance on the right side, in which $72(65 \%)$ out of 111 stents were inserted.

\section{Short-term outcome}

Successful re-opening of the airways was noted in all patients. $52(80 \%)$ out of 65 subjects experienced relieve of the clinical symptoms leading to stent insertion. Detailed pulmonary function tests were available in 61 patients. Mean increase in FEV1 and FVC was $299 \pm 531 \mathrm{~mL}(+21 \pm 33 \%$ compared to preinsertion) and $187 \pm 480 \mathrm{~mL}(+8 \pm 18 \%)$, respectively. Among the early complications (prior to day 30), symptomatic stent obstruction due to tenacious secretions (mucus plugging) was noted in seven patients, along with stent migration requiring removal (on day 1 and 3 ) in two patients. There was no procedure-related mortality.

\section{Late complications}

Study subjects had 15 (0-47) visits to our out-patient clinic after stent insertion; the first visit was a median 20 days after insertion; follow-up was 777 (7-3655) days. Two patients were lost to follow-up after 7 and 77 days, respectively.

Overall stent-related complications were seen in 52 (80\%) out of 65 patients. Among the late complications, re-stenosis occurred most frequently $(n=34)$, with new bacterial colonisation being second $(n=26)$. Isolated pathogens are listed in table 2. 13 subjects were colonised with Aspergillus sp. Further observed late complications were stent fracture $(n=6)$, haemoptysis $(n=7)$ and atelectasis $(n=3)$. Haemoptysis was successfully treated by topical administration of haemostyptic agents or argon coagulation in six out of seven cases. One patient died from fatal bleeding 128 days after stent insertion and 301 days after leftsided lung transplantation. An autopsy revealed focal invasive pulmonary aspergillosis of the graft without bleeding source at the stent site.

Re-stenosis occurred 85 (17-629) days after insertion. Restenosis occurred in $33(97 \%)$ out of 34 patients during the first 2 yrs after insertion (fig. 2). The most frequently used treatment modalities (including combinations chosen) were balloon dilatation $(n=21)$, argon coagulation $(n=18)$, cryotherapy $(n=2)$, brachytherapy $(n=12)$ and stent-in-stent insertion $(n=8)$. Besides the removal of two stents due to migration (both reinserted in a second intervention), no other late stent removal occurred. Risk factors for in-stent stenosis are shown in table 3. Only stent insertion before day 90 proved to be an independent covariate associated with re-stenosis.

\section{Outcome}

Graft loss (16 deaths and two redo-transplantations) was observed in 18 (27.6\%) patients after 727 (35-2.371) days. The most frequent causes of graft loss were BOS $(n=5)$, infection 


\begin{tabular}{lc}
\hline TABLE 2 Isolates in recipients with airway colonisation \\
\hline Airway colonisation before first stent \\
Pseudomonas aeruginosa & $17(26)$ \\
Staphlococcus aureus & $10(15)$ \\
Escherichia coli & $2(3)$ \\
Stenotrophomonas maltophilia & $4(6)$ \\
Achromobacter & $1(2)$ \\
Polymicrobial & $6(9)$ \\
Any & $29(45)$ \\
New airway colonisation after stent & \\
Pseudomonas aeruginosa & $17(26)$ \\
Staphlococcus aureus & $12(18)$ \\
Escherichia coli & $2(3)$ \\
Proteus mirabilis & $2(3)$ \\
Stenotrophomonas maltophilia & $1(2)$ \\
Achromobacter & $1(2)$ \\
Acinetobacter baumanii & $1(2)$ \\
Polymicrobial & $14(22)$ \\
Any & $26(40)$ \\
\hline Data are presented as n (\%). & \\
\hline
\end{tabular}

$(n=5)$, cardiovascular death $(n=3)$, respiratory failure $(n=2)$, haemoptysis $(n=1)$, malignancy $(n=1)$ and unknown causes $(n=1) .27(41.5 \%)$ patients developed BOS 853 (104-1848) days after stent insertion. Overall survival was significantly lower in lung transplant recipients with SEMS compared to the total cohort during the study period (fig. 3; $\log$ rank $p=0.02$ ), while BOS-free survival was not affected $(\mathrm{p}=0.27)$.

\section{DISCUSSION}

In this study, we present the largest cohort of lung transplant recipients with airway complications after insertion of SEMS, with detailed long-term follow-up of a median 2 yrs. Stentrelated complications during follow-up were noted in $80 \%$ of study subjects with the leading complications being in-stent stenosis and airway colonisation. Long-term survival was impaired in patients with SEMS.

There have been previous studies reporting complications during follow-up of patients after SEMS insertion; however, most of these report on patients with malignant airway disease [9, 15-19], and lung transplant recipients were infrequently included [17, 19]. To our knowledge, five small case series involving up to 33 patients have been published on the exclusive use of SEMS in lung transplant recipients [7, 20-23].

The long-term complication rate in subjects with malignant airway disease treated with SEMS is not comparable with lung transplant recipients. In recently published studies follow-up was just 47 to 128 days and covered tracheal stents were used in the majority of patients $[9,16,18]$. In contrast, airway disease in lung transplantation mainly involves the bronchi.

SEMS have undergone technical evolution and the Ultraflex ${ }_{\mathbb{B}}$ stent (Boston Scientific) has been shown to have a lower rate of complications, such as mucus plugging, migration and longterm stability, compared with the first and second generation SEMS, and it has excellent adaptability [24]. Recently published case reports with Ultraflex ${ }_{\circledR}$ stents in lung transplant

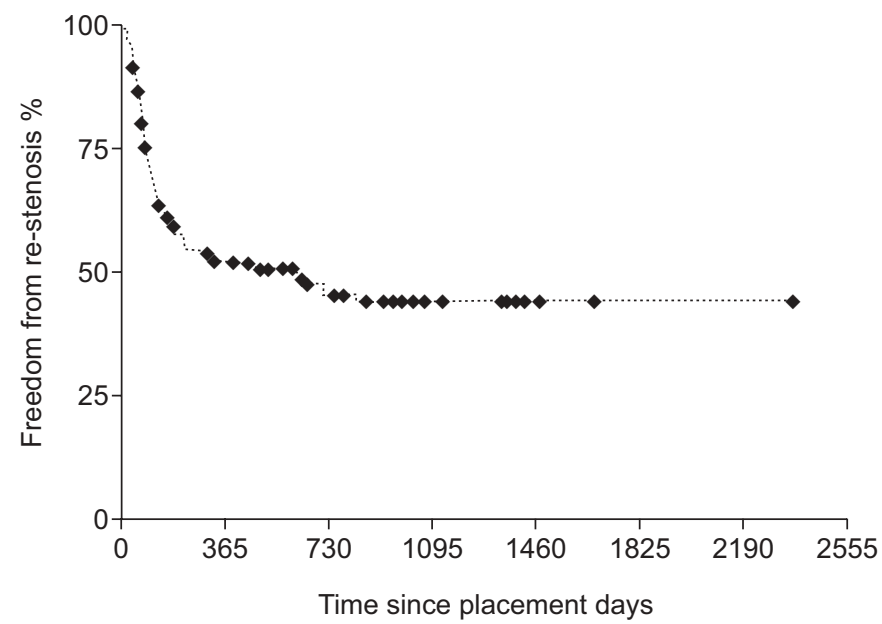

FIGURE 2. Kaplan-Meier plot of re-stenosis free survival after self-expandable metallic stents insertion in 65 lung transplant patients.

patients [20-22] included seven to 30 patients with a follow-up duration between 263 and 400 days, compared with 777 days in our study. Our first patient fitted with an Ultraflex ${ }_{\circledR}$ stent is still alive, $>10$ yrs after insertion and bronchoscopy performed 3.547 days after insertion revealed a patent stent lumen.

The vast majority of our SEMS recipients experienced some complication, appearing higher than the $22-67 \%$ reported in other studies $[9,16,18]$. This is explained by the longer followup period and the closer microbiological work-up. Microbiological data was not reported in most other studies.

Our re-stenosis rate was $52 \%$, which is higher than in other studies reporting a re-stenosis rate of $10-47 \%[9,17,18,20,21]$. This may be due to our longer follow-up period, given that restenosis occurred up to 2 yrs after insertion and that we used mainly noncovered stents. The higher re-stenosis rate when the stent was placed during the first three post-operative months can be explained by pronounced tissue inflammation in early lesions, which increases the risk of stent in-growth.

Lower airway tract colonisation is a common phenomenon in patients with impaired pulmonary defence and airway remodeling. Bacterial colonisation is frequent in lung transplant recipients with airway homeostasis and who are immunosuppressed. Two recent studies in unselected lung transplant recipients $[25,26]$ have described an incidence of Pseudomonas colonisation in 41 and $42 \%$, respectively, comparable to the $45 \%$ reported in our study before stent insertion. In contrast, $77 \%$ of our patients showed any bacterial airway colonisation after stenting, which is higher than expected.

NOPPEN et al. [27] reported an $80 \%$ incidence of bacterial colonisation (most frequently Staphylococcus aureus) after placement of silicone stents in nontransplant patients without prior colonisation, while bacterial airway colonisation decreased from $45 \%$ to $25 \%$ following therapeutic rigid bronchoscopy without stent placement [28]. BURNS et al. [21] studied colonisation in lung transplant patients with SEMS and demonstrated that $56 \%$ of BAL specimens were positive. Recent studies have shown that Pseudomonas colonisation of the allograft was associated with a higher risk for BOS. 
TABLE 3 Univariate and multivariate analysis (Cox proportional hazard model) of 65 recipients with self-expandable metallic stents for risk factors for stent re-stenosis

\begin{tabular}{ccccccc}
\multicolumn{2}{c}{ Univariate analysis } & & \multicolumn{3}{c}{ Multivariant analysis } \\
\cline { 1 - 1 } \cline { 5 - 6 } No re-stenosis & $\begin{array}{c}\text { Patients with stent } \\
\text { stenosis }\end{array}$ & p-value & & p-value & Adjusted HR & $95 \% \mathrm{Cl}$
\end{tabular}

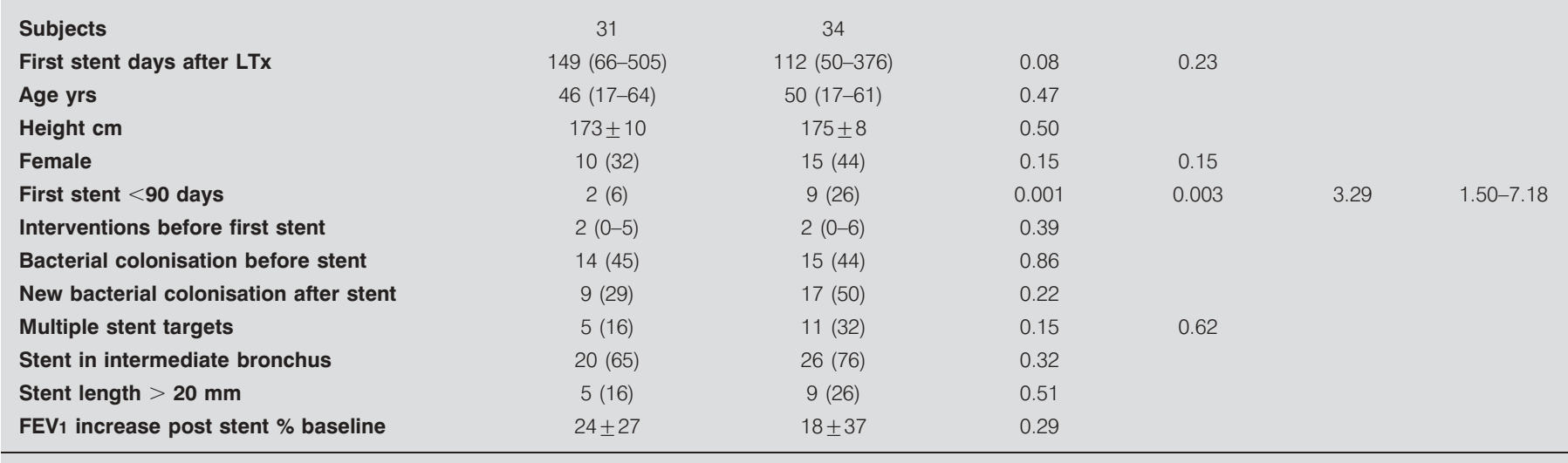

Data are presented as $\mathrm{n}$, median (range), mean $\pm \mathrm{SD}$ or $\mathrm{n}(\%)$, unless otherwise stated. LTx: lung transplantation; FEV1: forced expiratory volume in $1 \mathrm{~s}$.

BOS remains the major obstacle to long-term survival after lung transplantation $[25,26]$.

Clinical improvement rates and other observed complication rates (mucus plugging, migration and haemoptysis) were comparable to other studies using Ultraflex® stents $[9,16-18,21]$.

In our study, long-term survival in patients with SEMS was lower than in controls, which is comparable to the results of CHHAJED et al. [22]. Higher mortality in lung transplant recipients with SEMS may be explained by a higher risk of infection and lower functional reserve in case of complications. As in most other studies, we did not report any procedurerelated deaths [9, 15-20]. Even if the population with stents had a higher mortality than the population without stents, it is

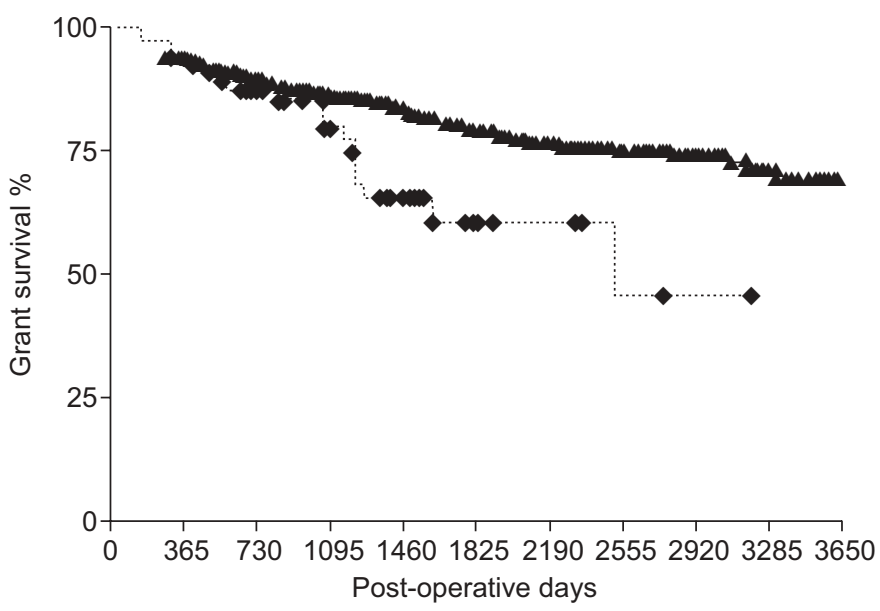

FIGURE 3. Kaplan-Meier survival comparing lung transplant patients with $(\bullet$ or without ( $\mathbf{\Lambda}$ ) self-expandable metallic stents (SEMS) from Hanover Medical School (Hanover, Germany) during the period 1998-2008. Only patients who survived for 90 days were included in the cohort of patients without SEMS. Log rank $p=0.02$. interesting to note that the difference in survival became apparent after $>3$ yrs. This may indicate the clinical significance of late complications. In $6 \%$ of our patients, death occurring $>30$ days after the proceedure was even potentially related to local complications, such as infection and haemoptysis.

The major limitation of this study is the lack of a control group and that it was a retrospective study. Airway colonisation and re-stenosis may occur even without SEMS. Desobliteration techniques have their own side-effects. Thermal desobliteration techniques may lead to deep tissue injury with secondary scarring, and repeat balloon dilatation may lead to tissue damage. To date, none of these have been compared with SEMS in a randomised controlled trial.

In some centres, implantation of prosthesis for a limited time is preferred [20] but removal of an epithelialised SEMS is difficult and potentially hazardous to the patient. LuNN et al. [29] reported on 30 stent extractions and found 32 complications, including mucosal tears, retained stent fragments and airway re-obstruction.

Theoretically, silicone stents are suitable as temporary prosthesis because they are easily removable and show no in-growth. In lung transplant recipients, silicone stents have not gained popularity because lesions are often complex, have a smaller inner diameter and frequently require ventilation through interstices. To date, no ideal airway stent exists that combines the advantages of silicone and metal stents. Biodegradable stents offer an exciting solution in the future: extraction is unnecessary, and the normal airway is preserved after stent resorption. In human studies in benign oesophageal strictures, these stents have shown to be clinically effective [30].

Successful prevention of airway complications seems to be a key issue of this often frustrating clinical problem. Surgical techniques to avoid excessive airway ischaemia and medical 
regimens to avoid fungal colonisation in the poorly vascularised post-anastomotic region have been proposed. It has been demonstrated recently that this standardised strategy resulted in a rate of just $4.9 \%$ of obstructive airway complications without the need of any airway stent in 235 patients over a $15-y r$ period [31].

In conclusion, SEMS were effective in the short-term management of benign airway disorders after lung transplantation but have a high complication rate in the long term. Indication of SEMS in lung transplant recipients should be critically discussed. The optimal treatment in this setting is unknown. Other therapies, such as airway debridement and balloon dilatation, should be considered prior to the placement of a SEMS. Permanent insertion of SEMS before post-operative day 90 should be strictly avoided. Removable or biodegradable stents may play a role in the future for this challenging problem.

\section{STATEMENT OF INTEREST}

None declared.

\section{ACKNOWLEDGEMENTS}

The authors would like to thank C. Schultheiss for data acquisition and M. Greer for revising the English in this manuscript (both Dept of Respiratory Medicine, Hanover Medical School, Hanover, Germany).

\section{REFERENCES}

1 Murthy SC, Blackstone EH, Gildea TR, et al. Impact of anastomotic airway complications after lung transplantation. Ann Thorac Surg 2007; 84: 401-409.

2 Herrera JM, McNeil KD, Higgins RS, et al. Airway complications after lung transplantation: treatment and long-term outcome. Ann Thorac Surg 2001; 71: 989-993.

3 Van De WC, Van Raemdonck D, Verleden GM, et al. Risk factors for airway complications within the first year after lung transplantation. Eur J Cardiothorac Surg 2007; 31: 703-710.

4 Chhajed PN, Malouf MA, Glanville AR. Bronchoscopic dilatation in the management of benign (non-transplant) tracheobronchial stenosis. Intern Med J 2001; 31: 512-516.

5 Keller CA, Hinerman R, Singh A, et al. The use of endoscopic argon plasma coagulation in airway complications after solid organ transplantation. Chest 2001; 119: 1968-1975.

6 Halkos ME, Godette KD, Lawrence EC, et al. High dose rate brachytherapy in the management of lung transplant airway stenosis. Ann Thorac Surg 2003; 76: 381-384.

7 Carre P, Rousseau H, Lombart L, et al. Balloon dilatation and selfexpanding metal Wallstent insertion. For management of bronchostenosis following lung transplantation. The Toulouse Lung Transplantation Group. Chest 1994; 105: 343-348.

8 Bolliger CT, Mathur PN, Beamis JF, et al. ERS/ATS statement on interventional pulmonology. European Respiratory Society/ American Thoracic Society. Eur Respir J 2002; 19: 356-373.

9 Breitenbucher A, Chhajed PN, Brutsche $\mathrm{MH}$, et al. Long-term follow-up and survival after Ultraflex stent insertion in the management of complex malignant airway stenoses. Respiration 2008; 75: 443-449.

10 Lund ME, Force S. Airway stenting for patients with benign airway disease and the Food and Drug Administration advisory: a call for restraint. Chest 2007; 132: 1107-1108.
11 Becker HD. Stenting of central airways. J Bronchol 1995; 2: 98-106.

12 Herth F, Becker HD, LoCicero J III, et al. Successful bronchoscopic placement of tracheobronchial stents without fluoroscopy. Chest 2001; 119: 1910-1912.

13 Miller MR, Hankinson J, Brusasco V, et al. Standardisation of spirometry. Eur Respir J 2005; 26: 319-338.

14 Estenne M, Maurer JR, Boehler A, et al. Bronchiolitis obliterans syndrome 2001: an update of the diagnostic criteria. J Heart Lung Transplant 2002; 21: 297-310.

15 Hautmann H, Bauer M, Pfeifer KJ, et al. Flexible bronchoscopy: a safe method for metal stent implantation in bronchial disease. Ann Thorac Surg 2000; 69: 398-401.

16 Husain SA, Finch D, Ahmed M, et al. Long-term follow-up of ultraflex metallic stents in benign and malignant central airway obstruction. Ann Thorac Surg 2007; 83: 1251-1256.

17 Madden BP, Park JE, Sheth A. Medium-term follow-up after deployment of ultraflex expandable metallic stents to manage endobronchial pathology. Ann Thorac Surg 2004; 78: 1898-1902.

18 Miyazawa T, Yamakido M, Ikeda S, et al. Implantation of ultraflex nitinol stents in malignant tracheobronchial stenoses. Chest 2000; 118: 959-965.

19 Saad CP, Murthy S, Krizmanich G, et al. Self-expandable metallic airway stents and flexible bronchoscopy: long-term outcomes analysis. Chest 2003; 124: 1993-1999.

20 Mughal MM, Gildea TR, Murthy S, et al. Short-term deployment of self-expanding metallic stents facilitates healing of bronchial dehiscence. Am J Respir Crit Care Med 2005; 172: 768-771.

21 Burns KE, Orons PD, Dauber JH, et al. Endobronchial metallic stent placement for airway complications after lung transplantation: longitudinal results. Ann Thorac Surg 2002; 74: 1934-1941.

22 Chhajed PN, Malouf MA, Tamm M, et al. Ultraflex stents for the management of airway complications in lung transplant recipients. Respirology 2003; 8: 59-64.

23 Kapoor BS, May B, Panu N, et al. Endobronchial stent placement for the management of airway complications after lung transplantation. J Vasc Interv Radiol 2007; 18: 629-632.

24 Freitag L, Eicker K, Donovan T, et al. Mechanical properties of airway stents. J Bronchology 1995; 2: 270-278.

25 Botha P, Archer L, Anderson RL, et al. Pseudomonas aeruginosa colonization of the allograft after lung transplantation and the risk of bronchiolitis obliterans syndrome. Transplantation 2008; 85: 771-774.

26 Vos R, Vanaudenaerde BM, Geudens N, et al. Pseudomonal airway colonisation: risk factor for bronchiolitis obliterans syndrome after lung transplantation? Eur Respir J 2008; 31: 1037-1045.

27 Noppen M, Pierard D, Meysman M, et al. Bacterial colonization of central airways after stenting. Am J Respir Crit Care Med 1999; 160: 672-677.

28 Noppen M, Pierard D, Meysman M, et al. Absence of bacterial colonization of the airways after therapeutic rigid bronchoscopy without stenting. Eur Respir J 2000; 16: 1147-1151.

29 Lunn W, Feller-Kopman D, Wahidi M, et al. Endoscopic removal of metallic airway stents. Chest 2005; 127: 2106-2112.

30 Saito Y, Tanaka T, Andoh A, et al. Usefulness of biodegradable stents constructed of poly-l-lactic acid monofilaments in patients with benign esophageal stenosis. World J Gastroenterol 2007; 13: 3977-3980.

31 Weder W, Inci I, Korom S, et al. Airway complications after lung transplantation: risk factors, prevention and outcome. Eur J Cardiothorac Surg 2009; 35: 293-298. 